\title{
Reactor Pulse Operation for Nuclear Instrumentation Detector Testing - Preparation of a Dedicated Experimental Campaign at the JSI TRIGA Reactor
}

\author{
Vladimir Radulović ${ }^{1}$, Loïc Barbot ${ }^{2}$, Grégoire De Izarra ${ }^{2}$, Julijan Peric ${ }^{3}$ and Igor Lengar ${ }^{1}$ \\ ${ }^{1}$ Jožef Stefan Institute, Reactor Physics Division, Slovenia \\ ${ }^{2}$ CEA, DES, IRESNE, DER, Instrumentation Sensors and Dosimetry Laboratory, Cadarache, F-13108 \\ Saint Paul-lez-Durance, France \\ ${ }^{3}$ University of Ljubljana, Faculty of Mathematics and Physics, Slovenia \\ vladimir.radulovic@ijs.si
}

\begin{abstract}
The availability of neutron fields with a high neutron flux, suitable for irradiation testing of nuclear instrumentation detectors relevant for applications in nuclear facilities such as material testing reactors (MTRs), nuclear power reactors and future fusion reactors is becoming increasingly limited. Over the last several years there has been increased interest in the experimental capabilities of the $250 \mathrm{~kW}$ Jožef Stefan Institute (JSI) TRIGA research reactor for such applications, however, the maximal achievable neutron flux in steady-state operation mode falls short of MTR-relevant conditions. The JSI TRIGA reactor can also operate in pulse mode, with a maximal achievable peak power of approximately $1 \mathrm{GW}$, for a duration of a few ms. A collaboration project between the JSI and the French Atomic and Alternative Energy Commission (CEA) was initiated to investigate absolute neutron flux measurements at very high neutron flux levels in reactor pulse operation. Such measurements will be made possible by special CEA-developed miniature fission chambers and modern data acquisition systems, supported by the JSI TRIGA instrumentation and activation dosimetry. Additionally, measurements of the intensity of Cherenkov light are proposed and being investigated as an alternative experimental method. This paper presents the preparatory activities for an exhaustive experimental campaign, which were carried out in 2019-2020, consisting of test measurements with not fully appropriate fission chambers, activation dosimetry and silicon photomultipliers (SiPMs) The presented results provide useful and promising experimental indications relevant for the design of the experimental campaign.
\end{abstract}

Keywords - Reactor Pulse, Fission Chamber, Activation Dosimetry, Cherenkov Light, Silicon Photomultiplier.

\section{INTRODUCTION}

$\mathrm{T}$ HE availability of neutron fields with a high neutron flux, suitable for irradiation testing of nuclear instrumentation detectors (such as fission and ionization chambers and selfpowered neutron detectors) relevant for applications in nuclear facilities such as material testing reactors (MTRs), nuclear power reactors and future fusion reactors is becoming increasingly limited. Over the last several years there has been increased interest in the experimental capabilities of the $250 \mathrm{~kW}$ Jožef Stefan Institute (JSI) TRIGA research reactor for such applications. This is thanks to extensive experimental and computational efforts in the past to characterize the experimental conditions in the reactor, in large part in collaboration with the Instrumentation, Sensors and Dosimetry Laboratory (LDCI) of the French Atomic and Alternative Energy Commission (CEA) - Cadarache. The maximal achievable neutron flux in the reactor in steady-state operation mode is approximately $2 \times 10^{13} \mathrm{n} \mathrm{cm}^{-2} \mathrm{~s}^{-1}$, in contrast to the MTR-relevant neutron flux range $10^{14}-10^{15} \mathrm{n} \mathrm{cm}^{-2} \mathrm{~s}^{-1}$. But the JSI TRIGA reactor can also operate in pulse mode, where one of the reactor control rods is ejected from the reactor core, thus introducing a sufficiently large reactivity to achieve prompt supercriticality. This operation mode is made possible by the TRIGA fuel, in the form of a dispersion of uranium and zirconium hydride, which gives rise to a large, prompt and negative temperature coefficient of reactivity. The resulting time dependence of the reactor power is a pulse, the peak power and duration depending on the initial inserted reactivity. In pulse mode, the maximal achievable peak power is approximately $1 \mathrm{GW}$, corresponding to a peak neutron flux level of the order of $10^{17} \mathrm{n} \mathrm{cm}^{-2} \mathrm{~s}^{-1}$, for a duration of a few milliseconds.

Up to now pulse operation mode at the JSI TRIGA reactor has been investigated mainly in the academic context, for the validation and improvement of the Fuchs-Hansen model [1-6] describing the reactor behaviour during reactor pulses and for educational activities, e.g. in the framework of practical courses for students of the University of Ljubljana or dedicated courses, to be offered in the framework of the ENEEP platform [7], however its application for irradiation experiments was of limited scope. Recognizing the potential of reactor pulse mode for nuclear instrumentation detector testing, in particular the 
possibility of extending the useful neutron flux range up to MTR-relevant levels, in 2019, a bilateral collaboration project between the CEA and JSI was initiated. The aim of the project is the performance of absolute neutron flux measurements at very high neutron flux levels in reactor pulse operation. The measurements will be made possible by special CEA-developed miniature fission chambers (fissile deposit mass targeted at 50 ng) and by modern, validated, wide dynamic range data acquisition systems, in particular the CEA-developed MONACO system. In addition to the JSI TRIGA nuclear instrumentation, providing information on the peak power and released energy, activation dosimetry will be employed as a reference for the normalization of the recorded detector signals (neutron flux integral) for reactor pulses with differing characteristics. Finally, as an alternative experimental method enabling the measurement of the reactor power dependence during pulse operation, measurements of the intensity of Cherenkov light are proposed and being investigated.

This paper presents the 2019-2020 preparatory activities for an exhaustive experimental campaign to be carried out at the JSI TRIGA reactor jointly by CEA and JSI researchers in 2021. A series of test measurements using not fully appropriate fission chambers in reactor pulse operation was performed using a Keithley electrometer and the MONACO fission chamber acquisition system. Activation dosimetry measurements were performed for several thermal and fast neutron sensitive nuclear reactions. Photodiodes and silicon photomultipliers (SiPMs) have been tested in steady state and pulse operation modes. The presented results provide useful and promising experimental indications relevant for the design of the experimental campaign.

\section{FISSION CHAMBER MEASUREMENTS}

The LDCI Lab has been manufacturing specific miniature fission chambers with MI cable for more than two decades at the CEA Cadarache fission chamber (FC) workshop. The FCs are supported with some unique calibration methods [8] also developed at CEA, enabling absolute neutron flux measurements.

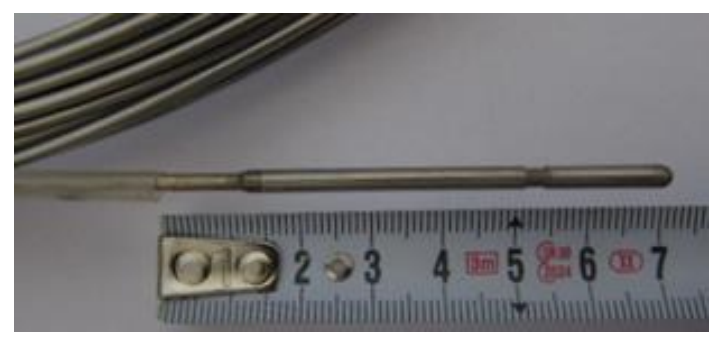

Fig. 1. CEA Cadarache manufactured miniature fission chamber.

In the last nine years, CEA developed a state-of-the art electronic acquisition system, named MONACO, which stands for 'Multichannel Online Acquisition in Campbell mOde'. In its current implementation, the system enables FC acquisition in pulse mode (i.e. counting pulses due to ionizations in the active gas, caused by energetic fission products), and in variance, or Campbell, mode (applicable when pulses are piling up, the signal variance being proportional to the incoming neutron flux) and current mode (pulse number become so high that their individual current are forming a measurable DC current proportional to the neutron and gamma environment). This implementation has been validated through numerous dedicated experimental campaigns, including several campaigns in the JSI TRIGA reactor [9, 10]. The current implementation (i.e. MONACOv2), now at TRL7 level, will be used in this project; the commercial version being currently industrialized in collaboration with the INSTRUMENTATION TECHNOLOGIES Company [11]. For this project, efforts will also be directed into increasing the acquisition rate of the MONACO2 system with a target of 1000 readings per second, enabling satisfactory discrimination of reactor pulses.

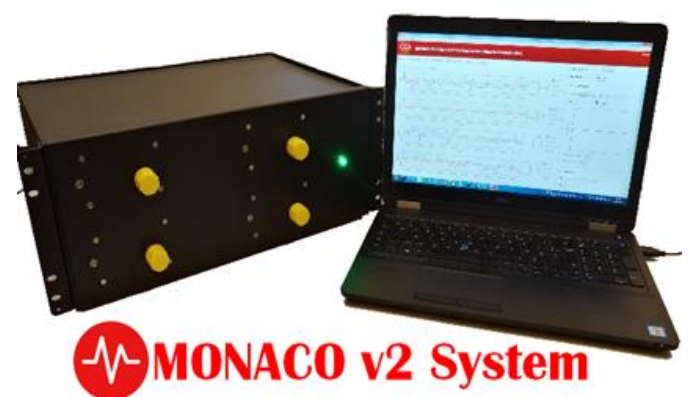

Fig. 2. CEA developed MONACOv2 acquisition system for fission chambers.

In preparation of the project experimental campaign, measurements in steady state and reactor pulse mode were performed in September 2019 using two existing CEA miniature fission chambers, with $\sim 10 \mu \mathrm{g}{ }^{235} \mathrm{U}$ fissile deposit. The FCs had an outer diameter of $3 \mathrm{~mm}$, and were positioned in an aluminium guide tube, located within Measurement Positions (MPs) in the lattice of the JSI TRIGA reactor core. A schematic view of the reactor core, a guide tube and a fission chamber is presented in Fig. 3.

Measurements were made using the MONACOv2 acquisition system [12]. A sequence of reactor pulses was carried out with varying inserted reactivity. Table 1 reports the reactor pulse operation parameters, as obtained from the JSI TRIGA reactor command control instrumentation: the pulse ID number, the prompt reactivity, the peak power, the full width at half maximum (FWHM) and the released energy.

TABLE I

PULSE PARAMETERS OBTAINED FROM THE JSI TRIGA INSTRUMENTATION

\begin{tabular}{ccccc}
\hline \hline Pulse ID & $\begin{array}{c}\rho_{\text {prompt }} \\
{[\$]}\end{array}$ & $\begin{array}{c}\text { Peak P } \\
{[\mathrm{MW}]}\end{array}$ & $\begin{array}{c}\text { FWHM } \\
{[\mathrm{ms}]}\end{array}$ & $\begin{array}{c}\text { Rel. E. } \\
{[\mathrm{MWs}]}\end{array}$ \\
\hline 360 & 1,10 & 1,0 & $503^{*}$ & 0,1 \\
361 & 1,15 & 1,3 & $6471^{*}$ & 0,1 \\
362 & 1,20 & 1,9 & $3818^{*}$ & 0,2 \\
363 & 1,25 & 3,3 & $2378^{*}$ & 0,3 \\
364 & 1,30 & 6,0 & 267 & 0,5 \\
365 & 1,35 & 10,3 & 166 & 0,9 \\
366 & 1,40 & 16,7 & 119 & 1,4 \\
367 & 1,40 & 20,0 & 106 & 1,6 \\
368 & 1,40 & 19,9 & 107 & 1,6 \\
369 & 2,00 & 240,2 & 27 & 7,5 \\
\hline \hline
\end{tabular}

*FWHM values obtained from the TRIGA instrumentation for low peak power pulses are not reliable. 

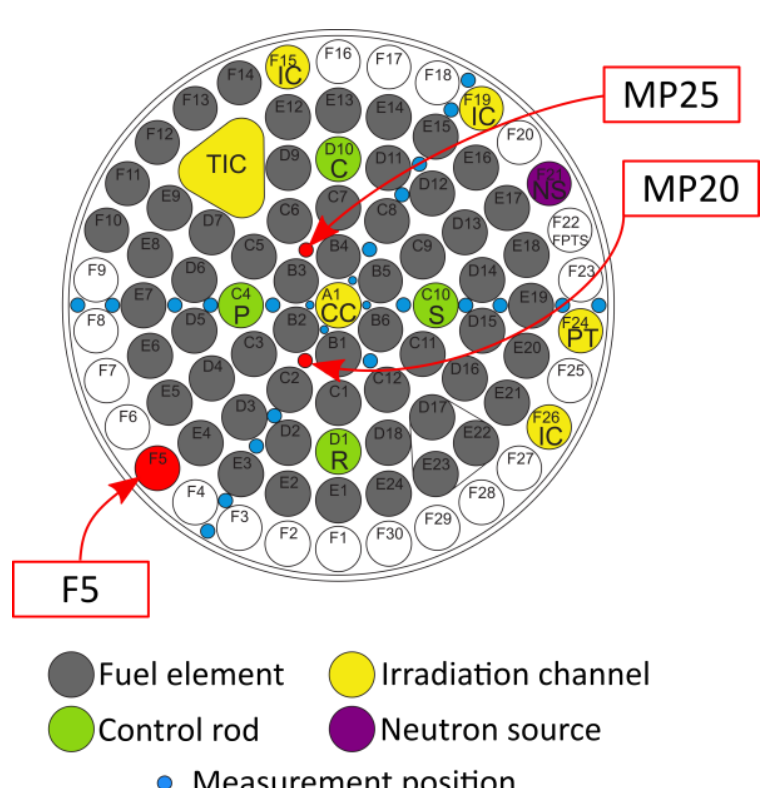

- Measurement position

\section{Core configuration (top view)}

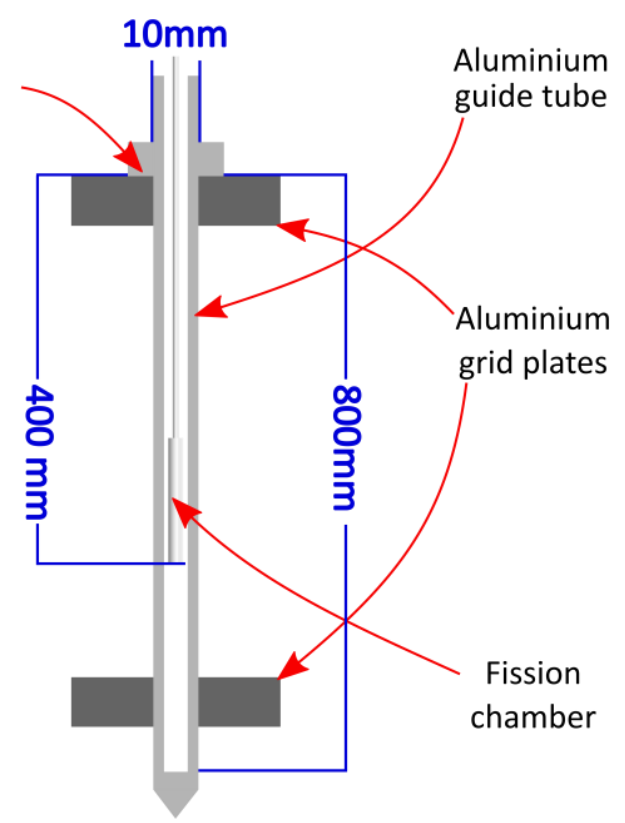

\section{Guide tube and FC (side view)}

Fig. 3. Top: Schematic view of the JSI TRIGA reactor core configuration (top view). Bottom: Schematic view of a fission chamber and a guide tube located between the reactor support plates.

The signal acquisition rates for the MONACO system varied approximately from 10 to 1000 samples s${ }^{-1}$. The measured pulse signals are displayed in Figs. 4 and 5.
FC signal variance vs. time, $1.2 \$$ pulse

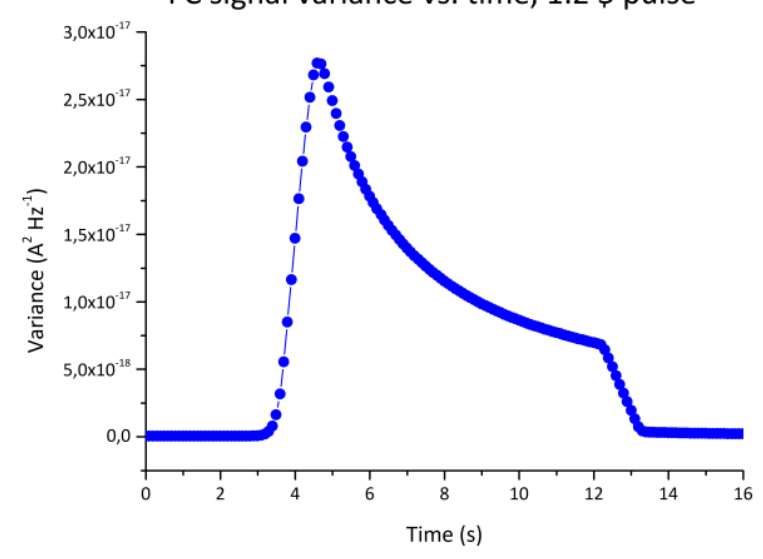

Fig. 4. Measured FC signals during a $1.2 \$$ reactor pulse. ${ }^{235} \mathrm{U}$ FC inserted in the core at the fuel mid plane.

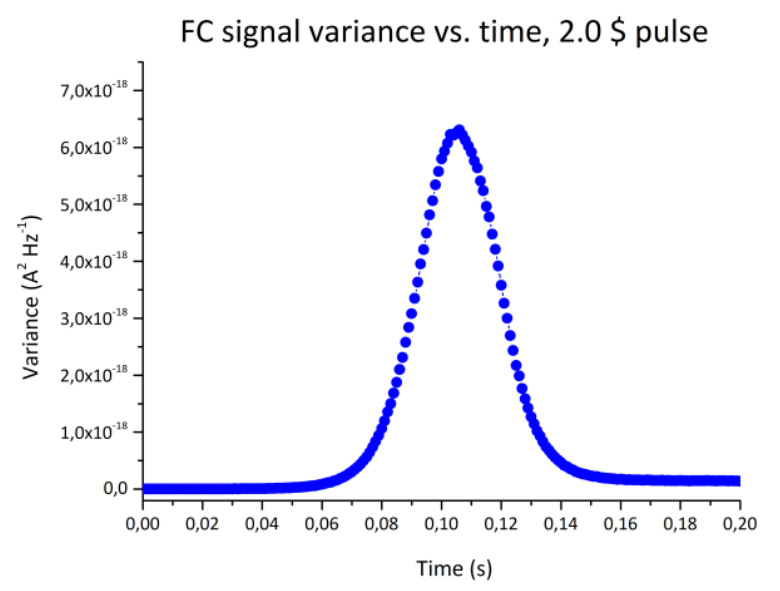

Fig. 5. Measured FC signals during a $2 \$$ reactor pulse. ${ }^{235} \mathrm{U}$ FC placed $\sim 200 \mathrm{~mm}$ above the core.

Despite the fact that $10 \mu \mathrm{g}{ }^{235} \mathrm{U}$ fissile deposits were not optimal for such measurements, the preliminary tests show a very satisfactory linearity between the maximal FC signals and the measured pulse peak power, as shown in Fig. 6.

Maximal FC signal variance vs. pulse peak power

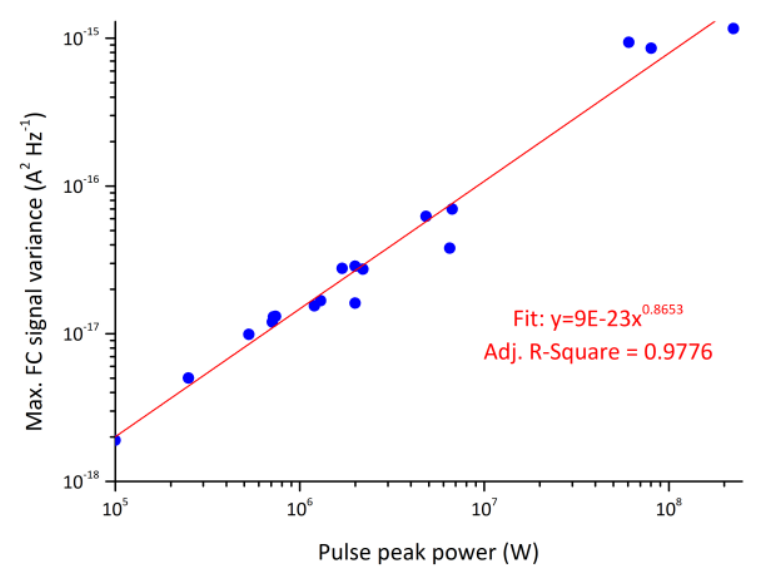

Fig. 6. Preliminary linearity of ${ }^{238} \mathrm{U} F \mathrm{~F}$ signals versus reactor peak power. 
Strong efforts will also be devoted for the final experimental campaign to optimize FC fissile deposits to high reactor power to perform absolute neutron flux measurements. Thanks to a series of three fine chemical analysis, target fissile masses of ${ }^{235} \mathrm{U}$ and ${ }^{238} \mathrm{U}$ are planned down to $<50 \mathrm{ng}$.

\section{ACTIVATION DOSIMETRY MEASUREMENTS}

Neutron activation dosimetry is the reference method in the determination of the neutron flux or fluence, and are included in the present work as a reliable experimental technique to obtain measurements of the total neutron fluence which can be delivered in pulse operation mode to samples of interest (e.g. neutron detectors, etc.). A series of test irradiations was performed in reactor pulse mode in which samples of certified reference materials $\mathrm{Al}-0.1 \% \mathrm{Au}, \mathrm{Al}-0.1 \% \mathrm{Co}$ and pure $\mathrm{Ni}$ were irradiated within polyethylene capsules in the Central Channel (CC) of the JSI TRIGA reactor. The induced activities in the samples were subsequently measured using two absolutely calibrated High-Purity Germanium (HPGe) detectors.

Fig. 7 displays the measured specific end-of-irradiation (EOI) activities (in Bq per $\mathrm{mg}$ of target material - e.g. per mg of $\mathrm{Co}$ in $\mathrm{Al}-0.1 \% \mathrm{Co}$ ) vs. the pulse prompt reactivity. The induced activities are proportional to the total neutron fluence received during the irradiations, which is in turn proportional to the released energy in the pulse. According to the FuchsHansen model [1] for reactor pulse operation, the energy released in a pulse is proportional to the prompt reactivity. The observed dependences clearly exhibit a linear behavior, taking into consideration the experimental uncertainties. In this work, these are relatively high (of the order of 3-4\%) in the context of reactor dosimetry measurements, on account of the low induced activities.

In the campaign, dosimetry measurements will serve as the reference for absolute normalization of the measured timedependent signals. In order to be able to complete a relatively high number of activation measurements during the experimental campaign (of the order of 100), the choice of optimal sample composition will be considered, enabling higher induced sample activities.

\section{Cherenkov Light MeAsurements}

As an alternative experimental method to record in detail the time dependence of the reactor power in real time, Cherenkov light measurements are considered. Cherenkov light is clearly visible in the reactor core during operation, especially in the outermost and mostly empty "F" ring, starting from approximately $10 \mathrm{~kW}$. To investigate this possibility, a measurement setup was created consisting of an aluminium channel (inside diameter approximately $34 \mathrm{~mm}$ ), closed on the bottom end and inserted into positon F4 in the reactor core. The channel had a slight curvature (approximately $0.5 \mathrm{~m}$ of side displacement between the top and bottom ends, $6 \mathrm{~m}$ apart) in order to limit neutron and gamma radiation streaming from the reactor core to the reactor platform. Approximately 1 liter of demineralized water was poured into the channel, serving as a
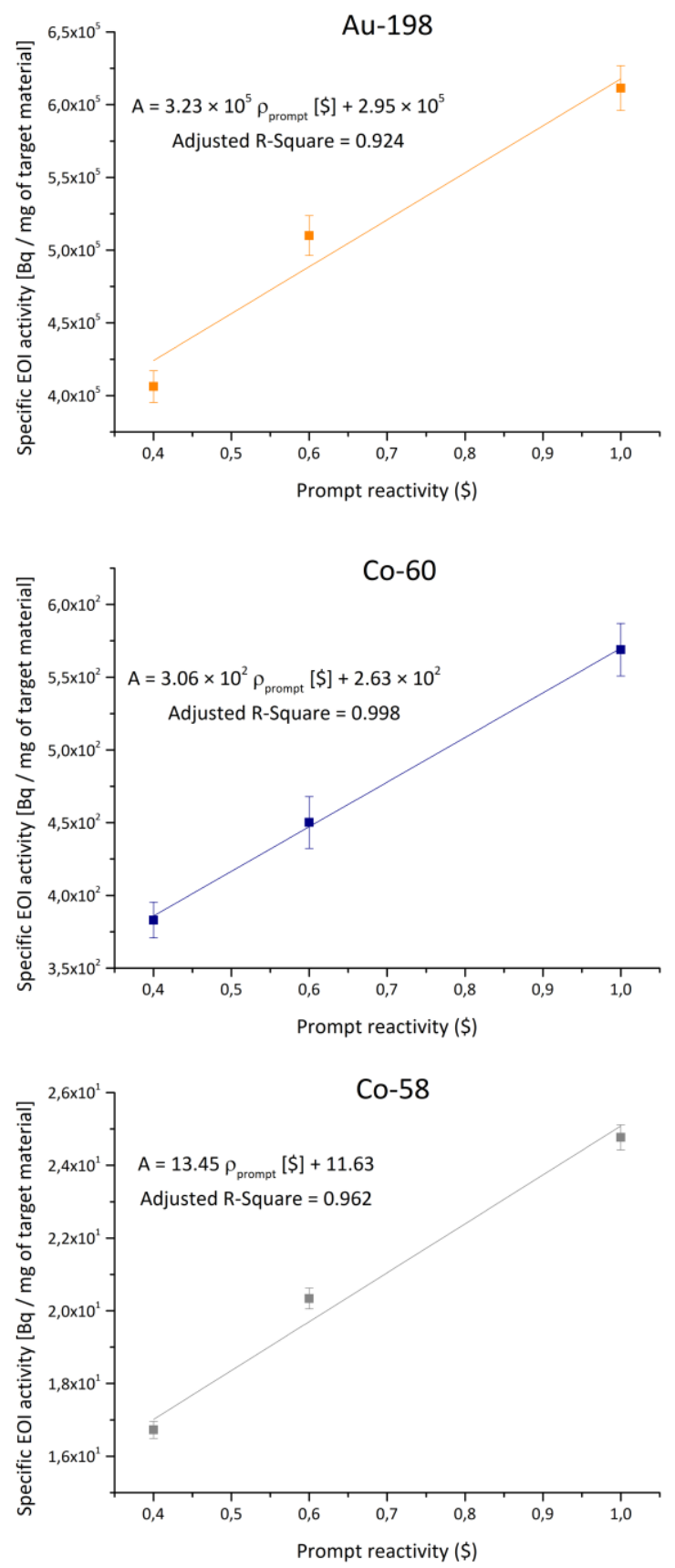

Fig. 7. Measured specific end-of-irradiation activities [Bq per $\mathrm{mg}$ of target material] for Au-198, Co-60 and Co-58, originating from the Au-197(n, $\gamma)$, Co$60(\mathrm{n}, \gamma)$ and $\mathrm{Ni}-58(\mathrm{n}, \mathrm{p})$ reactions, as a function of the prompt reactivity. A linear dependence is expected.

Cherenkov light radiator. The inner surface of the channel was smooth, which enabled the Cherenkov light produced in the water to be reflected and to be visible at the reactor platform level Fig. 8 a photograph of the setup on the JSI TRIGA reactor platform and Fig. 9 shows a photograph of the Cherenkov light inside the channel during reactor operation. 


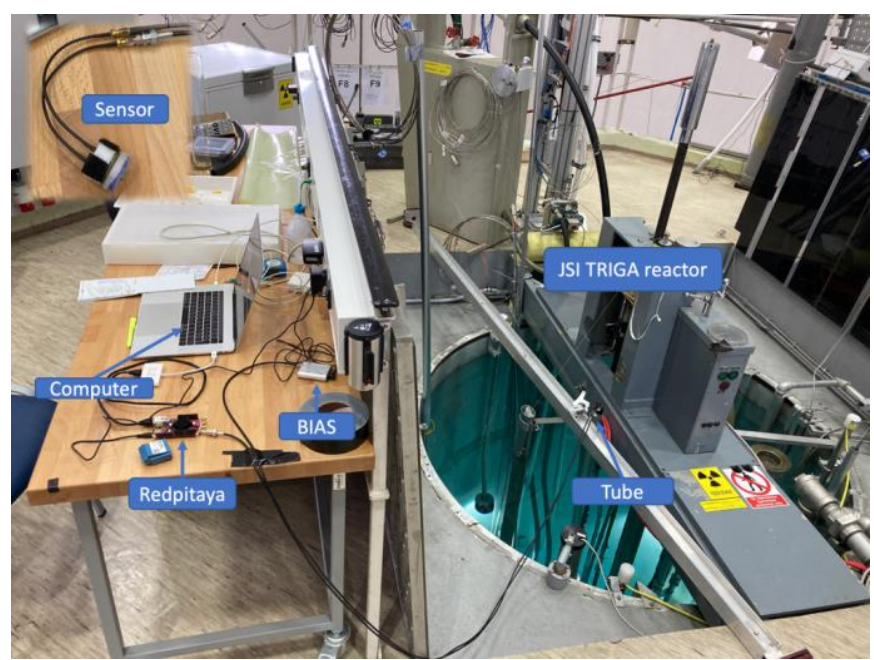

Fig. 8. Photograph of the Cherenkov light measurement setup on the JSI TRIGA reactor platform.

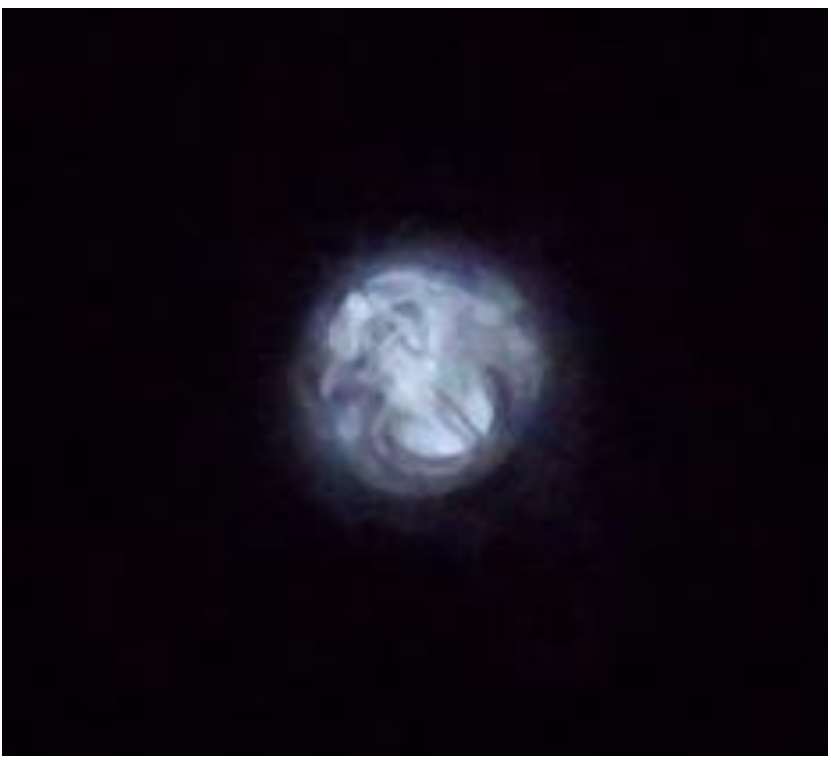

Fig. 9. Photograph of the actual Cherenkov light reaching the top of the tube during reactor operation.

The detector selected for light intensity measurements was a silicon photomultiplier (SiPM). Such detectors are nowadays inexpensive and are able to operate in single photon (pulse) mode as well as in current mode. The SiPM module used in the present work, along with the readout board, power supply were aquired from KETEK. A RedPitaya board [12] operated by a laptop computer was used to measure the voltage across the readout resistor in the SiPM readout board, proportional to the current flowing through the SiPM. A calibration of the SiPM detector was carried out in steady-state operation at several power steps from $2 \mathrm{~kW}$ to $250 \mathrm{~kW}$. Fig. 10 displays the measured SiPM signal with the power calibration applied and the reactor power in steady state operation, as a function of time, during the calibration phase. The SiPM signal dependence is clearly directly proportional to the reactor power, suggesting the feasibility of Cherenkov light measurements to obtain the time dependence of the reactor power during reactor pulses.

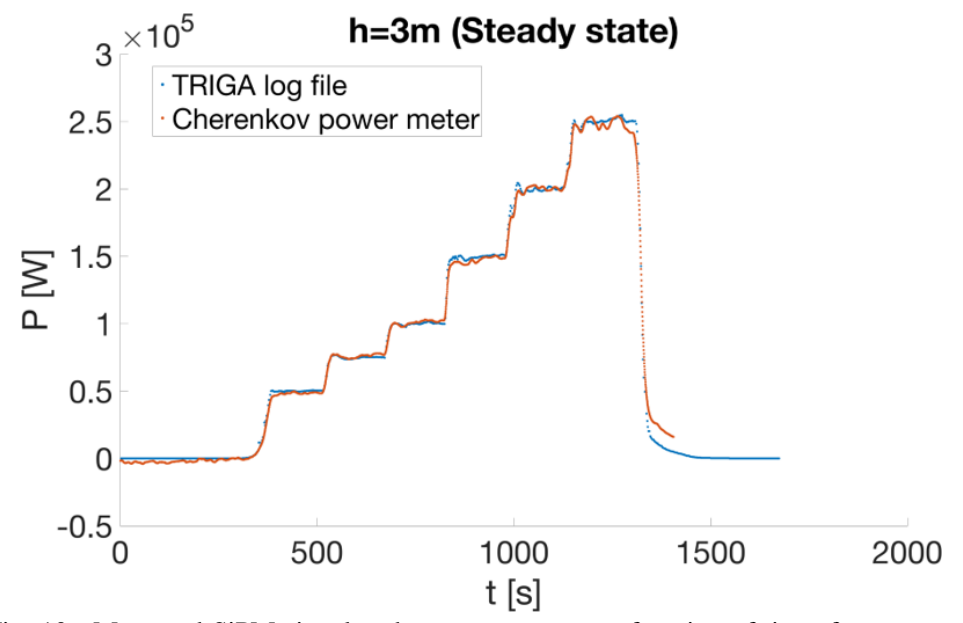

Fig. 10. Measured SiPM signal and reactor power as a function of time, for steady state reactor operation.

Subsequently, a series of pulses with increasing inserted reactivity was made and the SiPM signal was recorded. Fig. 11 displays the measured SiPM signals in reactor pulse mode, multiplied by the calibration factor determined in the calibration phase.

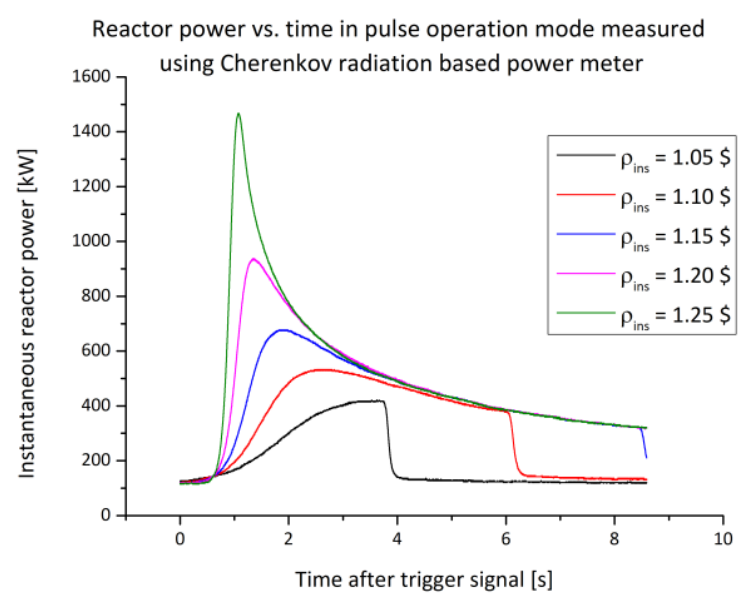

Fig. 11. Measured SiPM signal during reactor pulse operation, with power calibration factor applied.

A comparison of Figs. 4 and 11 (1.2 \$ pulse) shows that the measured FC and SiPM signals are qualitatively very similar, additionally confirming the applicability of the use of Cherenkov light measurements in reactor pulse mode.

\section{DISCUSSION}

The results obtained in the experimental activities presented in this work in preparation for a dedicated campaign demonstrate several important points. Miniature fission chambers in conjunction with the MONACO acquisition system perform well, in terms of their ability to measure a signal changing rapidly by several orders of magnitude. The measurements in the present work were performed in fission chamber variance mode. However, pulse acquisition mode (rapid counting of individual pulses, generated by fission events) is considered for the experimental campaign, as this operation mode enables more 
reliable absolute fission rate measurements and was used for benchmark fission rate measurements [13].

The obtained neutron dosimetry results demonstrate the feasibility of such measurements in reactor pulse mode for monitoring the total neutron fluence or the released energy. The EOI activities were relatively low (in the actual samples at most around $60 \mathrm{kBq}$ for $\mathrm{Au}-198$ in $\mathrm{Al}-0.1 \% \mathrm{Au}$ samples and at minimum $2 \mathrm{~Bq}$ for $\mathrm{Co}-60$ in $\mathrm{Al}-0.1 \% \mathrm{Co}$ samples. In the future experimental campaign, the low induced activities may be compensated to some degree by longer counting times in the gamma spectrometry measurements - however this may not be achievable for a large number of monitored pulses - and larger sample masses. In the present work, the material Al-0.1\% Co was used for the Co-59(n, $\gamma)$ reaction rate. To optimize the Co-60 activities for subsequent measurements, in the experimental campaign use will be made of Al-1\%Co, supplied by the CEA, as it is currently unavailable from the supplier.

The obtained results from the Cherenkov light intensity meter also clearly demonstrate its applicability for reactor pulse monitoring.

The experimental campaign will enable clear indications to support technological improvements in the JSI TRIGA pulse recorder, which was designed to cover a wide range of peak power levels, up to $2 \mathrm{GW}$, and consequently is not able to provide accurate information for low peak power pulses, which are deemed most interesting for instrumentation testing activities.

\section{CONCLUSIONS}

This paper presents the results of preparatory activities for a dedicated experimental campaign at the JSI TRIGA reactor on pulse operation. Measurements with miniature fission chambers and neutron dosimetry are of primary importance, and good results have been observed for both techniques. The results will also support required adjustments to further optimize the measurements, e.g. using longer counting times and higher sample masses for neutron dosimetry and extremely low fissile deposit masses in FCs using very accurate chemical analysis techniques.

Measurements of Cherenkov light were tested as an alternative and inexpensive technique to obtain experimental data on the pulse shapes. A simple setup based on a SiPM detector was created, refined and tested with success. Its use is proposed for in the dedicated experimental campaign.

The campaign results will enhance the JSI TRIGA reactor experimental capabilities in the context of detector and instrumentation testing activities, research in radiation hardness and potentially enable the JSI TRIGA reactor to support research in new fields or applications.

\section{ACKNOWLEDGMENT}

The authors acknowledge the financial support from the Slovenian Research Agency (research core funding No. P20073 - Reactor Physics, research project No. NC-0011 Absolute radiation measurements at very high neutron flux levels in reactor pulse mode).

\section{REFERENCES}

[1] G. I. Bell, S. Glasstone, Nuclear reactor theory, Van Nostrand Reinhold Company, New York, 1970.

[2] M. Ravnik, "Experimental Verification of Adiabatic Fuchs-Hansen Pulse Model", $4^{\text {th }}$ Regional Meeting Nuclear Energy in Central Europe, September 7-10, 1997, Bled, Slovenia.

[3] A. Pungerčič, I. Vavtar, L. Snoj, "Analysis of the JSI TRIGA pulse experiments”, in Proc. RRFM 2018, Munich, Germany, 2018.

[4] I. Vavtar, A. Pungerčič, L. Snoj, "Utilisation of JSI TRIGA pulse experiments for testing of nuclear instrumentation and validation of transient models", in Proc. ANIMMA 2019, Portorož, Slovenia, 2019. (EPJ Web of Conferences, ISSN 2100-014X, Vol. 225, 2020).

[5] I. Vavtar, L. Snoj, "Evaluation of approximations in reactor pulse", in Proc. PHYSOR 2020, Cambridge, UK, 2020.

[6] I. Vavtar, D. Ćalić, A. Pungerčič, L. Snoj, "Evaluation of approximation and uncertainties in theoretical models describing pulse mode operation" in Proc. RRFM 2020.

[7] M. Cagnazzo, H. Boeck, Š. Čerba, "The European Nuclear Experimental Educational Platform (ENEEP) for education and training", Atw Internationale Zeitschrift fuer Kernenergie, 65(5), 2020, 251-256.

[8] B. Geslot, T.C. Unruh, P. Filliatre, C. Jammes, J. Di Salvo, S. Bréaud, and J-F. Villard, "Method to Calibrate Fission Chambers in Campbelling Mode", IEEE Transactions on Nuclear Science (Volume: 59, Issue: 4, Aug. 2012).

[9] L. Barbot et al., "MONACO v2: Multipurpose and Integrated Data Acquisition System for On-line Neutron and Gamma Measurements", in Proc. ANIMMA 2019, Portorož, Slovenia, 2019 (EPJ Web of Conferences, ISSN 2100-014X, Vol. 225, 2020).

[10] Y. Moline et al., "MONACO: a Case Study for a New System On Chip and Stream based Architecture for Nuclear Instrumentation", Proc. ANIMMA 2019, Portorož, Slovenia, 2019 (EPJ Web of Conferences, ISSN 2100-014X, Vol. 225, 2020).

[11] L. Barbot, G. De Izarra, C. Destouches, M. Cargnelutti, S. Zorzut and D. Bisiach, "Development of a wideband current amplifier dedicated to Fission Chamber measurement", to be presented at the 7th ANIMMA Conference, Prague, June 21-25, 2021.

[12] https://www.redpitaya.com/

[13] Ž. Štancar, L. Snoj, and L. Barbot, "Reaction Rate Distribution Experiments at the Slovenian JSI TRIGA Mark II Research Reactor, TRIGA-FUND-RESR-002," in International Hand-book of Evaluated Reactor Physics Benchmark Experiments.

Paris:NEA/NSC/DOC(2006)1, OECD NEA, 2017. 